\title{
The endometrium in adenomyosis
}

\section{Giuseppe Benagiano \& Ivo Brosens*}

Eutopic and ectopic endometria of women with adenomyosis show a series of metabolic and molecular abnormalities that increase angiogenesis and proliferation, decrease apoptosis, allow local production of estrogens, create progesterone resistance, and impair cytokine expression. These changes enhance the ability of the endometrium to infiltrate the junctional zone myometrium and the growth of ectopic tissue. In addition, in these subjects several immunological abnormalities have been observed, together with an increased production of 'free radicals' leading to excessive growth of endometrial stromal cells that may facilitate the establishment of adenomyosis. A limiting factor is that these studies have been performed on hysterectomy specimens representing final stages of the disease. This increased knowledge has created new therapeutic options, including the block of local aromatase production through the use of selective estrogen receptor modulators, estrogen-progestin combinations and gonadotropin-releasing hormone super agonists. Also promising are investigations into the mechanism of dysmenorrhea and abnormal uterine bleeding.

Over the last 20 years, the scientific community devoted increasing attention to structural, as well as functional, changes occurring in endometrial differentiation, metabolism, apoptosis, neo-angiogenesis and innervation in women with endometriosis, suggesting a primary role for a higher than physiological invasiveness of eutopic endometrium in the genesis of the disease [1-4].

Although to a lesser extent, a number of investigations have also addressed the issue of the existence of changes occurring in eutopic endometrium in women with adenomyosis. In this case, however, attention has also been drawn to alterations observed in the inner myometrium, specifically an increase in its thickness [5]. This increased knowledge has important consequences as it is leading to new therapeutic options, both in terms of pain relief, including dysmenorrhea and abnormal uterine bleeding as well as providing the basis for treatment with adenomyosis-associated infertility.

A number of hypotheses have been proposed to explain the genesis of adenomyosis. At the same time, discussing the etiology of the condition is outside the scope of this review, as in this article the focus will be on experimental data, not hypotheses. Therefore, the specific aim of this review is to describe how a modified endometrium with an increased 'aggressiveness' may represent one of the mechanisms through which adenomyosis may be produced. In addition, although it is true that a hypothesis may be formulated on the fact that depending on the 'aggressiveness' of the modified endometrium, the disease may progress to a different extent, but there is insufficient information available to elaborate on this point.
Here, we wish to summarize information gathered over the last two decades on changes in the endometrium and inner myometrium in women with adenomyosis. In doing so it is important to stress that - contrary to what happens in women with endometriosis - even after noninvasive diagnosis became possible, studies of the endometrium in adenomyosis patients have been invariably carried out on hysterectomy samples. This means that, as yet, no information exists on the endometrium in initial or subclinical forms of the disease; a further consequence is that no epidemiological data on the incidence of adenomyosis are available, although, back in 1995, de Souza et al. detected patterns of adenomyosis in 54\% of young women with infertility and dysmenorrhea or menorrhagia [6].

\section{Methodology}

For this review, we searched the database SCOPUS (from 1990 to the middle of 2011) for all full texts and/or abstract articles published in English. Search terms included 'uterine adenomyosis' and 'eutopic endometrium' and with or without 'ectopic endometrium'. In fact, the search of 'adenomyosis' and 'endometrium' revealed over 900 papers. The search of 'adenomyosis' and 'eutopic endometrium' resulted in 104 papers; of these articles we selected those showing changes that may facilitate the establishment of the disease. In addition, upon screening the results for applicable titles and/or abstracts - with one exception - only the articles with original data in the international English literature were utilized (Table 1). In total, 29 articles included data on the eutopic endometrium in women with uterine adenomyosis. Data on the ectopic endometrium were only occasionally reported. Articles

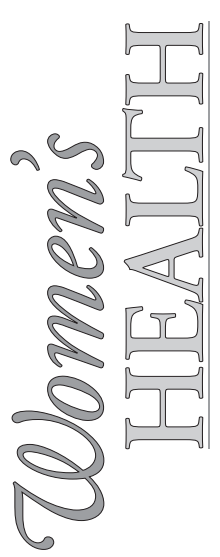

'Department of Gynecology, Obstetrics \& Urology, Sapienza, University of Rome, Rome, Italy

*Author for correspondence: Leuven Institute for Fertility \& Embryology, Leuven, Belgium ivo.brosens@med.kuleuven.ac.be

\section{Keywords}

- abnormal uterine bleeding

- adenomyosis • dysmenorrhea

- ectopic endometrium • eutopic endometrium $\bullet$ hysterectomy

- infertility $\bullet$ pathogenesis

\section{Future Medicine part of}


Table 1. Clinical data on studies of the eutopic endometrium in adenomyosis.

\begin{tabular}{|c|c|c|c|c|c|}
\hline Author (year) & Number of cases & Mean age (years) & Method of diagnosis & Groups & Ref. \\
\hline \multicolumn{6}{|l|}{ Innervation } \\
\hline Zhang et al. (2009) & 26 & 37 & Hysterectomy & $E, E$ and $A, F, C$ & [14] \\
\hline \multicolumn{6}{|l|}{ Angiogenesis } \\
\hline Qiu et al. (2006) & 133 (total) & & Hysterectomy & $\mathrm{E}, \mathrm{C}$ & [19] \\
\hline Li et al. (2006) & 68 & 32.5 & Hysterectomy & C & [20] \\
\hline Tokyol et al. (2009) & 25 & 47 & Hysterectomy & $E, C$ & {$[17]$} \\
\hline Goteri et al. (2009) & 14 & & Hysterectomy & C & [21] \\
\hline \multicolumn{6}{|c|}{ Proliferation and apoptosis } \\
\hline Matsumoto et al. (1999) & 23 & & Hysterectomy & C & [25] \\
\hline Yang et al. (2007) & 21 & & Hysterectomy & $C$ & [24] \\
\hline \multicolumn{6}{|c|}{ Steroid and cytokine production } \\
\hline Yamamoto et al. (1993) ${ }^{\dagger}$ & & & No clinical data & & [26] \\
\hline Lei et al. (1993) & 15 & & Hysterectomy & C & [27] \\
\hline Sotnikova et al. (2002) & 23 & & US, hysteroscopy, hysterectomy & C & [29] \\
\hline Nie et al. (2009) & 50 & 43 & Hysterectomy & C & [28] \\
\hline \multicolumn{6}{|l|}{ Immune components } \\
\hline Ota et al. (1993) ${ }^{\dagger}$ & 50 & & & $E, C$ & [30] \\
\hline Ota et al. $(1996)^{\dagger}$ & 23 & & & $E, C$ & {$[31]$} \\
\hline Chiang et al. (1997) & 7 & & Hysterectomy & $E, C$ & {$[32]$} \\
\hline Ota et al. $(1997)^{\dagger}$ & 22 & & & $E, C$ & {$[33]$} \\
\hline Ota et al. (1997) & 43 & & Hysterectomy, MRI & $E, C$ & {$[34]$} \\
\hline Propst et al. $(2002)^{\dagger}$ & 16 & & Hysterectomy & & {$[35]$} \\
\hline Wang et al. (2008) & 34 & 44 & Hysterectomy & C & [36] \\
\hline \multicolumn{6}{|l|}{ Reactive oxygen species } \\
\hline Ota et al. (1999) & 38 & 20 & Hysterectomy & $E, C$ & {$[40]$} \\
\hline Ota et al. (2000) & 34 & & Hysterectomy & $E, C$ & {$[37]$} \\
\hline Ota et al. (2001) & 34 & & Hysterectomy & $E, C$ & {$[38]$} \\
\hline Ota et al. (2001) & 33 & & Hysterectomy & $E, C$ & [39] \\
\hline Ota et al. (2002) & 36 & & Hysterectomy & $E, C$ & {$[41]$} \\
\hline \multicolumn{6}{|c|}{ Pathogenesis, clinical significance and potential markers } \\
\hline Maia et al. (2006) & 25 & & Hysteroscopic resection & $C$ & {$[44]$} \\
\hline Yang et al. (2006) & 24 & & Hysterectomy & C & {$[51]$} \\
\hline Chen et al. (2010) & 81 & & Hysterectomy & C & [45] \\
\hline Nie et al. (2010) & 50 & 43 & US and hysterectomy & $C, E$ & {$[47]$} \\
\hline Khan et al. (2010) & 35 & & US and MRI & $E, F$ & [49] \\
\hline Khan et al. (2010) & 35 & & US and MRI & $E, F$ & [50] \\
\hline Nie et al. (2011) & 50 & 42 & Hysterectomy & $\mathrm{C}$ & {$[56]$} \\
\hline Liu et al. (2011) & 50 & 43 & Hysterectomy & $E, F, C$ & {$[57]$} \\
\hline Hatok et al. $(2011)^{\dagger}$ & $101(\mathrm{~A}$ and $\mathrm{E})$ & & & $E, C$ & {$[46]$} \\
\hline
\end{tabular}


were included if it was clearly indicated how the diagnosis of adenomyosis was made and how a control group was established.

What follows is a detailed review of the literature on the subject, divided into sections covering all major aspects of the problem.

\section{Endometrial anomalies}

A number of anomalies have been found both in women with adenomyosis and in those with endometriosis. This should not be surprising given recent data indicating that up to $35 \%$ of women with frank endometriosis, the presence of adenomyosis can also be determined [7]. In 2005 , Kunz et al., studying women with adenomyosis, found that in a subset of their subjects the proportion of patients with both adenomyosis and endometriosis was as high as $70 \%$ [8]. In a subsequent study Bazot et al. observed a lower proportion [9]; nonetheless, some $27 \%$ of the women with endometriosis also had signs of adenomyosis; finally, Larsen $e t$ al. recently found that $34.6 \%$ of the subjects with endometriosis had adenomyosis, compared with $19.4 \%$ in the reference group and that $39.9 \%$ of the women with endometriosis had an irregular junctional zone compared with $22.5 \%$ in the reference group [7].

\section{Innervation}

In 2002, Quinn and Kirk studied patterns of uterine innervation in normal as well as abnormal uteri removed at hysterectomy for a variety of clinical conditions [10]. Histologically normal uteri from nulliparous or parous women showed similar patterns of innervation, namely the presence of nerve bundles at the endometrial-myometrial interface and in the subserosal layers. In contradistinction to this, uteri with adenomyosis showed large areas of myometrium without nerves and absence of nerves in the neurovascular bundles supplying these areas. Then, in 2007, Quinn described in greater detail the innervation of the uterus in women with adenomyosis [11]. He used a standard immunohistochemical staining utilizing the antiprotein gene product 9.5 (PGP9.5) and again compared two groups of uteri: one to be considered normal and the other with a histopathological diagnosis of adenomyosis. Normal innervation of the uterus included concentrations of nerves in the subserosal layers and at the endometrial-myometrial interface with sparse, neurovascular bundles distributed throughout the myometrial stroma. In contradistinction to this, in cases with adenomyosis, he found no nerves in areas with adenomyotic foci and an absence of nerves at the endometrial-myometrial nerve plexus. Quinn concluded that adenomyosis is associated with loss of nerve fibers at the endometrial-myometrial interface and absence of nerve fibers in the adenomyotic foci.

In relation to the endometrium, its innervation has also been reported in women with endometriosis $[12,13]$. Zhang et al. found nerve fibers in the uterus of women with pain who do not have endometriosis and, in an attempt to determine whether nerve fibers in the functional layer of the endometrium are caused by endometriosis itself or are more commonly found in women with pain, they analyzed endometrial tissues from 30 women with endometriosis, 40 women with adenomyosis, 41 women with uterine fibroids and 47 with both adenomyosis and endometriosis [14]. When women had any of these conditions, the presence of PGP9.5 and the neurofilament protein, both highly specific for nerve fibers, could be demonstrated only in women with pain symptoms. The density increased in the basal layer of the endometrium and myometrium significantly in women with pain. Neurofilament protein fibers were detected in the basal layer of the endometrium and in the myometrium.

Subsequently, the same group carried out a study of contiguous endometrial and myometrial tissues in 37 women with uterine fibroids and 29 women with adenomyosis [15] and, while confirming the detection of PGP9.5-immunoactive nerve fibers in the functional layer of the endometrium in women with pain, but not in those without pain, found no statistical differences between women with adenomyosis and uterine fibroids. These investigations suggest that PGP9.5-immunoactive nerve fibers appear in the endometrium and myometrium of women with painful adenomyosis and uterine fibroids may play a role in pain generation in these two disorders.

\section{Angiogenesis}

In order to survive and grow, endometrial cells and stroma must acquire a blood supply; indeed, during a menstrual cycle there are waves of active angiogenesis within the endometrium, with the development of arterioles and a capillary network, an event involving a spatially regulated process of vascular smooth cell differentiation, which is under the influence of a number of factors [16]. The activity of some of these factors in women with adenomyosis has now been investigated with interesting results. 
Of special interest are enzymes called matrix metalloproteinases (MMPs). They are expressed in the human endometrium as a consequence of cellular events during the menstrual cycle that require extracellular matrix remodeling [17]. In 1997, Bruner et al. developed an experimental model through which they were able to demonstrate that estrogen treatment of human endometrial tissue in organ culture maintains secretion of MMPs [18]. Suppressing metalloproteinase secretion in vitro using progesterone, or blocking enzyme activity with metalloproteinase inhibitors, stopped the formation of ectopic lesions in this experimental model. In 2006, Qiu et al. found that endometrium samples from women with and without adenomyosis showed similar expression of MMP-2, MMP-3 and of the tissue inhibitor of metalloproteinase-2 (TIMP-2), and that quantitative expression of MMP-2 mRNA, MMP-3 mRNA and TIMP-2 mRNA were significantly lower in eutopic endometrium from controls compared with ectopic endometrium from patients with adenomyosis [19]. In addition, during the proliferative phase, eutopic endometrium from controls showed significantly increased expression of MMP-2 mRNA compared with that in the secretory phase, suggesting that endometria of subjects with adenomyosis possesses more invasiveness. Given the possible role of MMPs in the evolution of adenomyosis, Li and coworkers evaluated the expression of MMP-2 and -9, together with that of VEGF, a major mediator of angiogenesis and vascular permeability, and of microvessel density (MVD) [20]. They studied both the eutopic and ectopic endometrium of subjects with adenomyosis and found a significantly greater activity than in a normal endometrium $(p<0.05)$. MVD was higher in ectopic than in eutopic endometria with or without adenomyosis $(\mathrm{p}<0.05)$. In adenomyosis a positive correlation was observed between VEGF expression and MMP-2 ( $\mathrm{p}<0.001)$, as well as MMP-9 expression ( $\mathrm{p}=0.002)$. A positive correlation was also found between MVD and MMP-2 ( $<<0.001)$ or MMP-9 expression $(p<0.001)$. It seems therefore, that the elevation of MMP-2 or -9 expression may represent an important factor in the development of the disease, contributing to the invasion of endometrial tissues into the myometrium and angiogenesis in adenomyotic implants. Tokyol et al. also obtained similar results with MMP-2 [17]. Subsequently, Goteri et al. compared, in the same woman, the expression of VEGF and HIF-1 $\alpha$ by ectopic versus eutopic endometria in women with adenomyosis and found that both were increased, particularly in epithelial cells [21]; Kang et al. investigated four VEGF polymorphic alleles and found significant differences between adenomyosis patients and a control group in the allele frequencies and genotype distributions [22]. It seems that the presence of two alleles of the $V E G F$ gene $(22578 A$ and $21154 A$ ) may significantly decrease the risk of adenomyosis, representing potentially protective factors for its development.

\section{Proliferation \& apoptosis}

Jones et al. studied apoptosis and bcl-2 in paired eutopic and ectopic endometria from women with endometriosis $(\mathrm{n}=30)$ or adenomyosis $(\mathrm{n}=15)$ and compared them with control endometria $(n=30)$ [23]. Apoptotic cells were rare in eutopic, ectopic and control endometria and there were no significant differences between subject groups, or between eutopic and ectopic endometria. In contrast to stromal $b c l-2$ expression in endometriosis, the expression in samples from adenomyosis patients remained at low levels and did not show significant cyclical variation. In another study, Yang et al., determined the percentage of annexin $\mathrm{V}(+) / 7$-amino-actinomycin $\mathrm{D}(+)$ in single endometrial stromal cells (ESCs) in eutopic endometrium and found that it was much lower in women with adenomyosis when compared with a control group [24]. ESCs from subjects with adenomyosis proliferated more rapidly than those of the control group, whether they were cultured alone or were treated with estradiol, medroxyprogesterone acetate, IL- 6 or IFN- $\gamma$. It seems, therefore, that altered apoptosis and proliferation of the eutopic endometrium could possibly play a role in the pathophysiology of adenomyosis.

With the aim of determining whether adenomyotic lesions originate in the basal layer of the eutopic endometrium, Matsumoto et al. evaluated biological and proliferative properties of adenomyotic lesions [25]. They observed that in the eutopic endometrium, apoptosis was most frequently observed in epithelial cells during mid-to-late secretory phases; in addition, $b c l-2$ gene expression inversely correlated with the appearance of apoptosis. A similar tendency was observed in stromal cells. Finally, they observed that the ectopic endometrium in adenomyosis is rarely influenced by hormonal changes and has different biological and proliferative properties than the eutopic endometrium, concluding that adenomyotic lesions do not originate in the basal endometrium. 


\section{Steroid \& cytokine production $\&$ responsiveness}

In 1993 Yamamoto and coworkers detected aromatase and estrone sulfatase activity in glandular cells of eutopic and ectopic endometrial tissues in women with adenomyosis and concluded that newly synthesized estrogens may enhance the growth of ectopic tissue [26]. In the same year, Lei et al. demonstrated on hysterectomy specimens that invading endometrial glands in adenomyosis selectively express more human chorionic gonadotrophin/luteinizing hormone receptor mRNA and immunoreactive receptor protein than noninvading glands [27].

In contradistinction to this, compared with a normal endometrium, progesterone receptor isoform B (PR-B) and I $\mathrm{B} \alpha$ - a member of a family of cellular proteins that function to inhibit the NF- $\kappa \mathrm{B}$, a protein complex that controls the transcription of DNA - were statistically significantly reduced in ectopic as well as eutopic endometrium from women with adenomyosis [28].

Sotnikova et al. have suggested a significant role of local cytokine production impairment in the development of adenomyosis; they studied cytokine synthesis by mononuclear cells infiltrating the eutopic and ectopic endometrium and found that levels of IFN- $\gamma$ and $-\alpha$, TNF- $\alpha$, IL-1 $\beta$ and EGF in supernatants of eutopic endometrial mononuclear cells of women with adenomyosis were significantly increased, whereas the content of IL- 8 was reduced compared with that of the controls [29].

\section{Immune components}

As early as 1993, immunological abnormalities were identified in women with adenomyosis [30]; in affected subjects expression of the HLA-DR antigen in the glandular cells of both eutopic and ectopic endometria was significantly lower than in samples from normal controls. Ota and his group have further evaluated $\mathrm{T}$ cell subsets, including $\gamma \delta \mathrm{T}$ cells, HLA antigens and adhesion molecules expressed in endometria of patients with adenomyosis. Compared with samples from normal subjects, in both eutopic and ectopic endometria they found a significantly increased expression of these antigens, particularly in the number of $\gamma \delta \mathrm{T}$ cells in the stroma and adhesion molecules and HLA antigens on the glandular cells [31]. Subsequently, Chiang and Hill described key immune response elements in eutopic and ectopic endometria obtained at hysterectomy and observed that activated $\mathrm{T}$ cells, IFN- $\gamma$ and upregulation of antigen presentation may play a role in normal endometrial physiology [32]. T cells, IFN- $\gamma$ and HLA-DR-positive stromal cells were more abundant in the secretory endometrium than in proliferative samples. CD3, IFN- $\gamma$ and HLA-DR-positive cells were scattered throughout the myometrium and, interestingly, were concentrated around vessels. They concluded that the increased number of $\mathrm{T}$ cells, the higher expression of IFN- $\gamma$, and an enhanced antigen presentation in ectopic, compared with eutopic, endometrium supports the concept that cellular immune activation is clearly involved in adenomyosis.

Ota and Tanaka have also investigated the expression of very late activation antigens (VLA2, 3, 4, 5, 6 and E-cadherin) and found that the expression of VLA-2, VLA-3 and E-cadherin was significantly increased throughout the menstrual cycle in samples from subjects with adenomyosis [33]. By contrast, the expression of VLA-4 in the adenomyosis group was significantly reduced in the secretory phase. In their view, results suggest the presence of a defective microenvironment in the eutopic endometrium of adenomyosis patients. A similar increase in expression of heat shock protein 27 was noted in the eutopic endometrium from patients with adenomyosis compared with controls, regardless of the menstrual phase [34].

A number of leukocyte anomalies have also been observed in the endometrium of patients suffering from adenomyosis. Propst $e t$ al. have documented that the ligand of the so-called granulocyte macrophage-colony stimulating factor, related to macrophage recruitment and activation, is overexpressed in women with adenomyosis, compared with other premenopausal women [35]; this suggests that the granulocyte macrophage-colony stimulating factor may play a role in increasing the levels of activated macrophages in women with adenomyosis. In addition, these women express HLA-G and this may explain how their endometrial cells can escape from the hosts' immunosurveillance and survive without being eliminated by the immune system [36].

\section{Altered production of reactive oxygen species}

An important area of investigation of endometria from women with adenomyosis is that related to the production of free radicals. These are intimately involved in the physiology of reproduction and enzymes that produce and eliminate various free radicals and are distributed throughout the body. These enzymes are believed to modulate 
concentrations of free radicals at an optimal level and maintain the body's homeostasis. Reactive oxygen species may modulate growth of endometrial stroma and, under pathologic conditions such as adenomyosis and endometriosis, increased oxidative stress, and the depletion of antioxidants may contribute to excessive growth of endometrial stromal cells. For this reason, it is useful to study this class of enzymes in pathologies involving the endometrium.

The first enzymic activity investigated in women with adenomyosis is glutathione peroxidase. Its expression on the surface of glandular epithelia during the menstrual cycle in fertile controls is weak in the early proliferative phase and gradually increases to become most marked in the early secretory phase, decreasing thereafter. In contradistinction to this, glutathione peroxidase expression in the eutopic endometrium in adenomyosis and endometriosis is persistently higher than in controls throughout the menstrual cycle, suggesting a pathological role in both disorders [37].

A second enzyme investigated is xanthine oxidase. Its expression in the glandular epithelium of normal subjects varies according to the menstrual phase, but in patients with adenomyosis this variation differs completely from that of controls; xanthine oxidase expression seems to be present in ectopic endometrial tissue in all cases. Mean nomogram levels in the glandular epithelium in adenomyosis tissue have been found to be as high as those in the early secretory phase in the eutopic endometrium. The authors concluded that aberrant expression of xanthine oxidase in eutopic and ectopic endometria appears to play a pathological role in endometriosis and adenomyosis [38].

COX-2 is a third enzyme examined in connection with adenomyosis. Recent experiments suggest that COX-2 antagonizes cell apoptosis, increases invasiveness and promotes angiogenesis. Ota et al. determined the distribution of COX-2 in eutopic and ectopic endometria in endometriosis and adenomyosis [39]. The evaluation nomogram scores in glandular epithelium and stromal cells in adenomyosis tissue were $2.2 \pm 0.1$ and $1.1 \pm 0.2$, respectively, approximately the same as that in the early-to-mid proliferative phases in the eutopic endometrium. On the other hand, there is no variation in its expression during the menstrual cycle; also, levels are lower than in women with endometriosis. The authors concluded that increased COX-2 expression in eutopic and ectopic endometria seemed strongly correlated with pathological abnormalities in both disorders.
Ota et al. studied superoxide dismutases (SOD) in the eutopic endometrium of women with adenomyosis or endometriosis [40]. The expression of $\mathrm{Cu}-\mathrm{Zn}-\mathrm{SOD}$ and Mn-SOD in control subjects varies with the stages of the menstrual cycle. By contrast, SOD was persistently overexpressed regardless of the menstrual cycle in patients with endometriosis and adenomyosis. The exaggerated expression of these enzymes suggests a crucial role for superoxide in infertility and/or miscarriage in these diseases.

Finally, Ota et al. investigated the expression of a fifth enzyme involved in the metabolism of free radicals, catalase, in eutopic and ectopic endometria in patients with endometriosis or adenomyosis [41]. Contrary to what happens in the glandular epithelium of a normal endometrium where expression fluctuates during the menstrual cycle, being lowest in the early proliferative phase and peaking in the late secretory phase, in women with adenomyosis, catalase scores did not vary in patients with adenomyosis, and scores in this group were consistently higher than those in patients with endometriosis throughout the cycle. Catalase expression was found in ectopic endometrial tissue in all groups. The mean score in the glandular epithelium in adenomyotic tissue was $2.6 \pm 0.2$. Levels in the glandular epithelium were almost as high as those in the mid-to-late secretory phase in the eutopic endometrium.

\section{Pathogenesis}

The advent of noninvasive techniques for the study of adenomyosis has helped our knowledge of the pathophysiology of this disease, documenting that it can be already present in women in their 30s. This increased knowledge has now produced new therapeutic options, both pharmacological and surgical $[42,43]$.

Given that adenomyosis is an estrogendependent disease, a first-line investigation dealt with counteracting their response to estrogen suppression. Maia et al. determined whether aromatase expression in the eutopic endometrium and in adenomyotic foci is affected by use of an oral contraceptive (OC) [44]. In a retrospective study of endometrial tissues obtained from patients with a histological diagnosis of adenomyosis who had used an OC containing gestodene plus ethinyl estradiol, they measured aromatase expression and found that it was expressed in $80 \%$ of the eutopic endometrial slices, mainly in the stromal cells, whereas it was mostly absent in adenomyotic tissue. Use of the gestodene-containing $\mathrm{OC}$ was effective 
in suppressing aromatase expression in both the eutopic and ectopic endometrium. Although the study, being retrospective, did not include analysis before OC use and after; the finding tends to support the use of OCs in women with initial forms of adenomyosis. Recently, Chen et al. hypothesized that estrogens may endow epithelial-mesenchymal transition (EMT) cells with migratory and invasive properties [45]. To prove their hypothesis they investigated whether EMT occurred in adenomyotic lesions and whether it correlated with serum $17 \beta$-estradiol levels. They found that in the epithelial component of adenomyotic lesions, vimentin expression was upregulated and E-cadherin expression was downregulated compared with the eutopic endometrium. In addition, in adenomyosis, serum $\mathrm{E}_{2}$ levels were negatively correlated with E-cadherin expression in the epithelial components of both eutopic and ectopic endometria, suggesting the involvement of estrogen-induced EMT. Furthermore, in estrogen receptor-positive Ishikawa endometrial epithelial cells, estrogens induced increased migration and invasion, and upregulation of the EMT regulator Slug. Finally, in ovariectomized severe combined immunodeficiency mice, xenotransplanted eutopic or ectopic endometria from adenomyosis patients showed estrogen dependence and that the effect of estrogen was suppressed by the selective estrogen receptor modulator, raloxifene. Interestingly, raloxifene also abrogated the effect of estrogens in women [45]. These data suggest that raloxifene may represent a potential therapeutic agent for adenomyosis patients.

\section{Clinical significance}

Recently, Hatok et al. suggested that in addition to clinical data the measurement of endometrial aromatase cytochrome P450 (CYP19) in adenomyosis patients could be of discriminative value in the prediction of estrogen dependency of the disease [46]. Additional leads came from Nie et al. who studied the expression and localization of the oxytocin receptor and of the so-called transient receptor potential vanilloid type-1 [47]. The latter is a nonselective cation channel that may be activated by a wide variety of exogenous and endogenous physical and chemical stimuli. Together with the cannabinoid type-1 receptors, it has been proposed as a metabotropic and ionotropic receptor, respectively, for two classes of endogenous polyunsaturated fatty acid amides, the acylethanolamides and the acyldopamides [48]. In women with adenomyosis, immunoreactivity of oxytocin receptor and transient receptor potential vanilloid type-1 were significantly increased in the ectopic endometrium and both were positively correlated with the severity of dysmenorrhea, being significant predicators for its severity. These findings may have a potential therapeutic application.

Several studies exist on the multifactorial effects of gonadotropin releasing hormone superagonists (GnRHa) on adenomyotic tissues. Khan et al. have investigated the pattern of changes in inflammatory reaction, microvessel density and apoptosis in biopsy specimens obtained from eutopic and ectopic endometria in women with adenomyosis following treatment with GnRHa and compared them with untreated patients [49]. The infiltration of CD68-positive Mvarphi (a macrophage marker) and Von Willebrand Factor-positive microvessel density were significantly decreased in the endometria of women with adenomyosis when treated with a GnRHa. Thus, GnRHa treatment for periods of 3-6 months was able to markedly reduce the inflammatory reaction and angiogenesis and to significantly induce apoptosis in tissues derived from women with adenomyosis. This provides further rationale, beyond the production of hypoestrogenism, for the use of superagonist analogs in adenomyosis, because all three mechanisms are involved and contribute to the survival of the ectopic tissue. The same group has also investigated the expression of GnRH receptors (GnRHRs) and the effect of an analog on the proliferation of cells derived from endometria of women with adenomyosis [50]. They found that types I and II GnRHRs mRNA and proteins were expressed in eutopic and ectopic endometria from women with adenomyosis. GnRHRs expression was the highest around the time of menstruation. Exogenous treatment with GnRHa significantly suppressed the proliferation of cells derived from eutopic as well as ectopic endometria. Here again, in addition to a hypoestrogenic effect, a direct antiproliferative effect of GnRHa may be involved in the regression of adenomyotic foci with consequent remission of clinical symptoms.

Progestins have been utilized in patients with endometriosis for decades, although the absence of diagnostic tools prevented their use in those with adenomyosis. It seems that this treatment may produce a series of complex changes and more information is required before a final conclusion can be reached. On the one hand, there is a certain degree of progesterone resistance in 
patients with adenomyosis; Yang et al. believe that IL-6 liberated by endometrial stromal cells may play a role in the survival of the ectopic endometrium [51]. Inhibiting IL-6 production would therefore make it more difficult for an ectopic focus to survive. To this aim, they incubated single ESCs with medroxyprogesterone acetate or danazol from the eutopic endometrium of women with or without adenomyosis and found that treatment produces a significant decrease of IL- 6 concentration in the supernatant, in the control group, but not in cells from patients with adenomyosis. Therefore, medroxyprogesterone acetate and danazol seem to have a decreased effect on the suppression of IL-6 liberated by ESCs in adenomyosis and this phenomenon may play a role in the formation of ectopic endometrial implants in adenomyosis.

In addition, in a recent study Mehasseb et al. have investigated the distribution of estrogen and progesterone receptor isoforms throughout the menstrual cycle in the endometrium of subjects with and without adenomyosis, concluding that estrogen receptor-B expression and the lack of PR expression are probably related to the development and/or progression of the disease [52]. Also, these data provide a possible explanation why in these patients there is a poor response of menstrual symptoms to progestational agents.

\section{Potential markers}

On the other hand, Nie et al. have now described for the first time that in ectopic endometrial tissue in adenomyosis the promoter of PR-B is hypermethylated [53]. This epigenetic aberration demonstrates that adenomyosis is also an epigenetic disease amenable to rectification by pharmacological agents.

Nie et al. have recently reported interesting further developments [54]. A first lead is the finding that immunoreactive nuclear proteins p65, p50 and p52 were statistically significantly increased in ectopic and eutopic endometria of women with adenomyosis. Nuclear p65 immunoreactivity was positively associated with heavier menses, and decreased PR-B and increased nuclear p65 immunoreactivity in the ectopic endometrium and were statistically significantly associated with the severity of dysmenorrhea in women with adenomyosis. This finding may be developed into a marker for adenomyosis.

A second option for a marker is based on the work of Shen et al. who found the presence in ovarian endometriomas of SLIT, a secreted protein that functions through the Roundabout (ROBO) receptor as a repellent for axon guidance and neuronal migration [55]. The SLIT/ROBO1 system seems to induce tumor angiogenesis and may have a potential role as a marker of recurrence in endometriomas. Following-up on this hypothesis, Nie et al. found that SLIT expression was statistically significantly higher in the ectopic endometrium of women with adenomyosis, while ROBO1 immunoreactivity and microvessel density level were statistically significantly higher in both eutopic and ectopic endometria of women with adenomyosis [56]. This particular marker may be utilized as an index of the severity of dysmenorrhea. Also related to dysmenorrhea is the finding that the so-called tissue factor (TF) is upregulated in endometriosis. Applying this knowledge to adenomyosis, Liu et al. investigated the expression and localization of TF in the eutopic and ectopic endometrium of women with the condition and found that TF immunoreactivity was significantly increased in both eutopic and ectopic endometria in cases of adenomyosis and such an elevation is associated with heavy menses and increased severity of dysmenorrhea [57].

Finally, Huang and colleagues have just published that tyrosine kinase receptor B ( TrkB) is expressed in the eutopic endometrium of women with adenomyosis and that the average level of TrkB protein and of TrkB mRNA in secretory endometrial samples of women with adenomyosis was significantly higher than that in controls $(p<0.01)$ [58]. The expression level of the TrkB protein was positively correlated with the serum CA125 ( $p=0.016)$ and with the intensity of dysmenorrhea $(\mathrm{p}=0.002)$. The authors concluded that measurement of $\operatorname{TrkB}$ in the eutopic endometrium may represent a marker of the progression of adenomyosis.

The fact that new imaging techniques allow an early detection of adenomyosis means that the previously described new knowledge can also be applied to infertility associated with the disease. Indeed, today a number of medical and surgical techniques are available to improve the chances of conception in younger women with adenomyosis [59]. Having said this, the fact remains that substantially more information is necessary on the situation existing in the eutopic endometrium at the early stages of the disease to formulate a meaningful hypothesis on its role in causing adenomyosis.

An interesting approach for a comparative diagnosis of adenomyosis versus fibroids has been suggested by Hever et al. who analyzed 
gene expression patterns of normal endometrium and myometrium and compared them to that in the two conditions [60]. They discovered that the four tissue types could be identified based on their gene expression profiles. They found that adenomyosis could be clustered most closely with the endometrium and uterine fibroids with myometrium. They also compared the gene expression profiles of adenomyosis and uterine fibroids and identified 471 differently expressed probe sets that may represent potential biomarkers for the differential diagnosis.

In conclusion, evidence is also accumulating that endometrial anomalies in adenomyosis and endometriosis indicate that both are phenotypes of a single disorder rather than two distinct disease entities [61] and a report comparing the eutopic endometrium in both conditions is in preparation. In addition, the high correlation between the presence of endometriosis and that of adenomyosis in first degree relatives is suggestive of a common origin [62].

\section{Future perspective}

Within the next 5 years we should witness a progressive switch in the diagnosis of adenomyosis from a posthysterectomy finding to an early, noninvasive procedure (3D transvaginal ultrasound and MRI) capable of identifying early stages of the disease.

\section{Executive summary}

\section{Endometrial anomalies}

- Adenomyosis is associated with loss of nerve fibers at the endometrial-myometrial interface and absence of nerve fibers in the adenomyotic foci.

- The elevation of matrix metalloproteinase-2 and -9 expression may contribute to invasion of endometrial tissues into the myometrium and increased angiogenesis in adenomyotic implants.

- Altered apoptosis and proliferation of the eutopic endometrium can possibly play a role in the pathophysiology of adenomyosis.

- Aromatase and estrone sulfatase activity in glandular cells of eutopic and ectopic endometrial tissues in women with adenomyosis may enhance the growth of ectopic tissue.

- Numerous immunological abnormalities have been identified in women with adenomyosis.

- Reactive oxygen species modulate growth of endometrial stroma and under pathologic conditions, such as adenomyosis and endometriosis, increased oxidative stress and depletion of antioxidants may contribute to excessive growth of endometrial stromal cells.

- Glutathione peroxidase expression in the eutopic endometrium in adenomyosis and endometriosis is persistently higher than in controls throughout the menstrual cycle.

- The exaggerated expression of these enzymes suggests a crucial role for superoxide in subfertility and/or miscarriage in adenomyosis and endometriosis.

\section{Pathogenesis}

- A retrospective study of endometrial tissues obtained from patients with adenomyosis showed that aromatase was expressed in $80 \%$ of the eutopic endometrial slices, mainly in the stromal cells, whereas it was mostly absent in adenomyotic tissue.

- Estrogens may endow epithelial-mesenchymal transition cells with migratory and invasive properties.

- The effect of estrogen is suppressed by the selective estrogen receptor modulator raloxifene suggesting that this compound may represent a potential therapeutic agent for adenomyosis patients.

\section{Clinical significance}

- The measurement of endometrial aromatase cytochrome P450 (CYP19) in adenomyosis patients could be of discriminative value in the prediction of the degree of estrogen dependency of the disease.

- Gonadotropin-releasing hormone superagonist treatment for periods of 3-6 months is able to markedly reduce the inflammatory reaction and angiogenesis and to significantly induce apoptosis in tissues derived from women with adenomyosis.

- In addition to a hypoestrogenic effect, a direct antiproliferative effect of gonadotropin releasing hormone superagonist may be involved in the regression of adenomyotic foci with consequent remission of clinical symptoms.

- Progestins have been utilized in patients with endometriosis for decades, although the absence of diagnostic tools prevented their use in those with adenomyosis. However, there is a degree of progesterone resistance in patients with adenomyosis and progestins' effect may be diminished.

- A recent study concluded that estrogen-B expression and the lack of progesterone expression are probably related to the development and/or progression of the disease.

\section{Potential markers}

- It seems that adenomyosis is an epigenetic disease amenable to rectification by pharmacological agents.

- Nuclear p65 may be developed into a marker for adenomyosis in women with heavier menstrual bleeding and women with dysmenorrhea.

- Tyrosine kinase receptor B is expressed in eutopic endometrium of women with adenomyosis and its measurement in eutopic endometrium may represent a marker of the progression of adenomyosis. 
An improved and earlier diagnosis will have a major impact on three fronts:

- First, in the presence of infertility it will be easier to diagnose the existence of adenomyosis as a cause or contributing factor;

- Second, the new techniques will allow large screenings and therefore make it possible to collect data on the epidemiology of the disease and its true impact on fertility;

- Third, availability of transvaginal ultrasound and MRI will dramatically improve the possibilities for treatment, whether pharmacological, surgical or combined. A number of procedures are already available and over the next 5-10 years these techniques will be applied more and more widely. Comparative studies will follow and the superiority of one method over another will be determined.

Within 5 years endometrial markers of adenomyosis will have been identified and tested and within 10 years they will be widely applied.

New treatment modalities will be developed based on the increased knowledge of the pathophysiology of the disease; it is anticipated that within 10 years these will be able to block the progression of adenomyosis, possibly avoiding surgery altogether.

The concomitant presence of adenomyosis and endometriosis will be evaluated in statistically valid ways; this will permit the collection of 'clean' epidemiological data in younger women and the study of homogeneous groups of women with endometriosis, adenomyosis or both conditions, versus nonaffected controls. These data are needed in order to find out whether or when adenomyosis becomes a progressive disease and design conservative treatment at an early stage.

In the final analysis these studies will be able to provide a definitive response to the hypothesis that adenomyosis and endometriosis are phenotypes of the same metabolic and molecular disorder.

\section{Financial \& competing interests disclosure}

The authors have no relevant affiliations or financial involvement with any organization or entity with a financial interest in or financial conflict with the subject matter or materials discussed in the manuscript. This includes employment, consultancies, honoraria, stock ownership or options, expert testimony, grants or patents received or pending, or royalties.

No writing assistance was utilized in the production of this manuscript.

\section{References}

Papers of special note have been highlighted as: - of interest

1. Vinatier D, Cosson M, Dufour P. Is endometriosis an endometrial disease? Eur. J. Obstet. Gynecol. Reprod. Biol. 91, 113-125 (2000).

2. Sharpe-Timms KL. Endometrial anomalies in women with endometriosis. Ann. N. Y. Acad. Sci. 943, 131-147 (2001).

3. Carvalho L, Podgaec S, Bellodi-Privato M, Falcone T, Abrao MS. Role of eutopic endometrium in pelvic endometriosis. J. Minim. Invasive Gynecol. 18, 419-427 (2011).

4. Brosens I, Brosens JJ, Benagiano G. The eutopic endometrium in endometriosis: are the changes of clinical significance? Repro. BioMed. Online doi:10.1016/j.rbmo.2012.01.022 (2012) (Epub ahead of print).

5. Brosens JJ, de Souza NM, Barker FG. Uterine junctional zone: function and disease. Lancet 346, 558-560 (1995).

6. de Souza NM, Brosens JJ, Schwieso JE, Paraschos T, Winston RM. The potential value of magnetic resonance imaging in infertility. Clin. Radiol. 50, 75-79 (1995).
7. Larsen SB, Lundorf E, Forman A,

Dueholm M. Adenomyosis and junctional zone changes in patients with endometriosis. Eur. J. Obstet. Gynecol. Reprod. Biol. 157, 206-211 (2011).

8. Kunz G, Beil D, Huppert P, Noe M, Kissler S, Leyendecker G. Adenomyosis in endometriosis - prevalence and impact on fertility. Evidence from magnetic resonance imaging. Hum. Reprod. 20, 2309-2316 (2005).

9. Bazot M, Fiori O, Darai E. Adenomyosis in endometriosis - prevalence and impact on fertility. Evidence from magnetic resonance imaging. Hum. Reprod. 21, 1101-1102 (2006).

10. Quinn MJ, Kirk N. Differences in uterine innervation at hysterectomy. Am. J. Obstet. Gynecol. 187, 1515-1519 (2002).

11. Quinn M. Uterine innervation in adenomyosis. J. Obstet. Gynaecol. 7, 287-291 (2007).

12. Tokushige N, Markham R, Russell P, Fraser IS. High density of small nerve fibres in the functional layer of the endometrium in women with endometriosis. Hum. Reprod. 21, 782-787 (2006).

13. Tokushige N, Markham R, Russell P, Fraser IS. Different types of small nerve fibres in eutopic endometrium and myometrium in women with endometriosis. Fertil. Steril. 88, 795-803 (2007).

14. Zhang X, Lu B, Huang X, Xu H, Zhou C, Lin $\mathrm{J}$. Endometrial nerve fibers in women with endometriosis, adenomyosis, and uterine fibroids. Fertil. Steril. 92, 1799-1801 (2009).

- Nerve fibers could be demonstrated only in women with pain symptoms.

15. Zhang X, Lu B, Huang X, Xu H, Zhou C, Lin $J$. Innervation of endometrium and myometrium in women with painful adenomyosis and uterine fibroids. Fertil. Steril. 94, 730-737 (2010).

16. Kohnen G, Campbell S, Jeffers MD, Cameron IT. Spatially regulated differentiation of endometrial vascular smooth muscle cells. Hum. Reprod. 15, 284-292 (2000).

17. Tokyol C, Aktepe F, Dilek FH, Sahin O, Arioz DT. Expression of cyclo-oxygenase-2 and matrix metalloproteinase-2 in adenomyosis and endometrial polyps and its correlation with angiogenesis. Int. J. Gynecol. Pathol. 28, 148-156 (2009).

18. Bruner KL, Matrisian LM, Rodgers WH, Gorstein F, Osteen KG. Suppression of matrix metalloproteinases inhibits establishment of ectopic lesions by human endometrium in nude mice. J. Clin. Invest. 99, 2851-2857 (1997). 
19. Qiu F, Gao XM, Luo GL et al. [Expression of matrix metalloproteinase and tissue inhibitor of metalloproteinase in adenomyosis.] Sichuan Da Xue Xue Bao Yi Xue Ban 37, 118-122 (2006).

20. Li T, Li YG, Pu DM. Matrix metalloproteinase-2 and -9 expression correlated with angiogenesis in human adenomyosis. Gynecol. Obstet. Invest. 62, 229-235 (2006).

21. Goteri G, Lucarini G, Montik N et al. Expression of vascular endothelial growth factor (VEGF), hypoxia inducible factor-1 $\alpha$ (HIF-1 $\alpha$ ), and microvessel density in endometrial tissue in women with adenomyosis. Int. J. Gynecol. Pathol. 28(2), 157-163 (2009).

22. Kang S, Zhao J, Liu Q, Zhou R, Wang N, Li $\mathrm{Y}$. Vascular endothelial growth factor gene polymorphisms are associated with the risk of developing adenomyosis. Environ. Mol. Mutagen. 50, 361-366 (2009).

23. Jones RK, Searle RF, Bulmer JN. Apoptosis and $b c l-2$ expression in normal human endometrium, endometriosis and adenomyosis. Hum. Reprod. 13, 3496-3502 (1998).

24. Yang JH, Wu MY, Chen CD, Chen MJ, Yang YS, Ho HN. Altered apoptosis and proliferation in endometrial stromal cells of women with adenomyosis. Hum. Reprod. 22, 945-952 (2007).

- Endometrial stromal cells from subjects with adenomyosis proliferated more rapidly than those of the control group.

25. Matsumoto Y, Iwasaka T, Yamasaki F, Sugimori H. Apoptosis and Ki-67 expression in adenomyotic lesions and in the corresponding eutopic endometrium. Obstet. Gynecol. 94, 71-77 (1999).

26. Yamamoto T, Noguchi T, Tamura T, Kitawaki J, Okada H. Evidence for estrogen synthesis in adenomyotic tissues. Am. J. Obstet. Gynecol. 169, 734-738 (1993).

- Aromatase and estrone sulfatase activity was detected in glandular cells of eutopic and ectopic endometrial tissues in women with adenomyosis.

27. Lei ZM, Rao CV, Lincoln SR, Ackermann DM. Increased expression of human chorionic gonadotropin/human luteinizing hormone receptors in adenomyosis. J. Clin. Endocrinol. Metab. 76, 763-768 (1993).

28. Nie J, Lu Y, Liu X, Guo SW. Immunoreactivity of progesterone receptor isoform $\mathrm{B}$, nuclear factor $\kappa \mathrm{B}$, and $\mathrm{I} \kappa \mathrm{B} \alpha$ in adenomyosis. Fertil. Steril. 92, 886-889 (2009).

- Findings strongly suggest that both progesterone receptor-B and NF- $\kappa B$ are potential targets in treating adenomyosis, at least for relieving dysmenorrhea and menorrhagia.

29. Sotnikova N, Antsiferova I, Malyshkina A. Cytokine network of eutopic and ectopic endometrium in women with adenomyosis. Am. J. Reprod. Immunol. 47, 251-255 (2002).

30. Ota H, Igarashi S. Expression of major histocompatibility complex class II antigen in endometriotic tissue in patients with endometriosis and adenomyosis. Fertil. Steril. 60, 834-838 (1993).

31. Ota H, Igarashi S, Tanaka T. Expression of g $\delta \mathrm{T}$ cells and adhesion molecules in endometriotic tissue in patients with endometriosis and adenomyosis. Am. J. Reprod. Immunol. 35, 477-482 (1996).

32. Chiang CM, Hill JA. Localization of T cells, interferon- $\gamma$ and HLA-DR in eutopic and ectopic human endometrium. Gynecol. Obstet. Invest. 43, 245-250 (1997).

33. Ota H, Tanaka T. Integrin, adhesion molecules in the endometrial glandular epithelium in patients with endometriosis or adenomyosis. J. Obstet. Gynaecol. Res. 23, 485-491 (1997).

34. Ota H, Igarashi S, Hatazawa J, Tanaka T. Distribution of heat shock proteins in eutopic and ectopic endometrium in endometriosis and adenomyosis. Fertil. Steril. 68, 23-28 (1997).

35. Propst AM, Quade BJ, Nowak RA, Stewart EA. Granulocyte macrophage colonystimulating factor in adenomyosis and autologous endometrium. J. Soc. Gynecol. Invest. 9, 93-97 (2002).

36. Wang F, Wen Z, Li H, Yang Z, Zhao X, Yao X. Human leukocyte antigen- $G$ is expressed by the eutopic and ectopic endometrium of adenomyosis. Fertil. Steril. 90, 1599-1604 (2008).

37. Ota H, Igarashi S, Kato N, Tanaka T. Aberrant expression of glutathione peroxidase in eutopic and ectopic endometrium in endometriosis and adenomyosis. Fertil. Steril. 74, 313-318 (2000).

38. Ota H, Igarashi S, Tanaka T. Xanthine oxidase in eutopic and ectopic endometrium in endometriosis and adenomyosis. Fertil. Steril. 75, 785-790 (2001).

39. Ota H, Igarashi S, Sasaki M, Tanaka T. Distribution of cyclooxygenase-2 in eutopic and ectopic endometrium in endometriosis and adenomyosis. Hum. Reprod. 16, 561-566 (2001).

40. Ota H, Igarashi S, Hatazawa J, Tanaka T. Immunohistochemical assessment of superoxide dismutase expression in the endometrium in endometriosis and adenomyosis. Fertil. Steril. 72, 129-134 (1999).

41. Ota H, Igarashi S, Sato N, Tanaka H, Tanaka $\mathrm{T}$. Involvement of catalase in the endometrium of patients with endometriosis and adenomyosis. Fertil. Steril. 78, 804-809 (2002).

42. Kitawaki J. Adenomyosis: the pathophysiology of an oestrogen-dependent disease. Best. Prac. Res. Clin. Obstet. Gynaecol. 20, 493-502 (2006).

43. Benagiano G, Brosens I, Carrara S. Adenomyosis: new knowledge is generating new treatment options. Womens Health (Lond. Engl.). 5, 297-311 (2009).

44. Maia H Jr, Casoy J, Correia T et al. Effect of the menstrual cycle and oral contraceptives on aromatase and cyclooxygenase- 2 expression in adenomyosis. Gynecol. Endocrinol. 22, 547-551 (2006).

45. Chen YJ, Li HY, Huang $\mathrm{CH}$ et al. Oestrogeninduced epithelial-mesenchymal transition of endometrial epithelial cells contributes to the development of adenomyosis J. Pathol. 222, 261-270 (2010).

- Estrogens endow epithelial-mesenchymal transition cells with migratory and invasive properties.

46. Hatok J, Zubor P, Galo S et al. Endometrial aromatase mRNA as a possible screening tool for advanced endometriosis and adenomyosis. Gynecol. Endocrinol. 27, 331-336 (2011).

47. Nie J, Liu X, Guo SW. Immunoreactivity of oxytocin receptor and transient receptor potential vanilloid type 1 and its correlation with dysmenorrhea in adenomyosis. Am. J. Obstet. Gynecol. 202(4), 346.e1-8 (2010).

48. Cristino L, de Petrocellis L, Pryce G, Baker D, Guglielmotti V, Di Marzo V.

Immunohistochemical localization of cannabinoid type 1 and vanilloid transient receptor potential vanilloid type 1 receptors in the mouse brain. Neuroscience 139, 1405-1415 (2006).

49. Khan KN, Kitajima M, Hiraki K et al. Changes in tissue inflammation, angiogenesis and apoptosis in endometriosis, adenomyosis and uterine myoma after GnRH agonist therapy. Hum. Reprod. 25, 642-653 (2010).

- Rationale for the use of superagonist analogs in adenomyosis.

50. Khan KN, Kitajima M, Hiraki K et al. Cell proliferation effect of GnRH agonist on pathological lesions of women with endometriosis, adenomyosis and uterine myoma. Hum. Reprod. 25, 2878-2890 (2010).

- First paper based on adenomyosis diagnosed by noninvasive techniques.

51. Yang JH, Chen MJ, Wu MY, Chen YC, Yang YS, Ho HN. Decreased suppression of interleukin- 6 after treatment with medroxyprogesterone acetate and danazol in 
endometrial stromal cells of women with adenomyosis. Fertil. Steril. 86, 1459-1465 (2006).

52. Mehasseb MK, Panchal R, Taylor AH, Brown L, Bell SC, Habiba M. Estrogen and progesterone receptor isoform distribution through the menstrual cycle in uteri with and without adenomyosis. Fertil. Steril. 95, 2228-2235 (2011)

53. Nie J, Liu X, Guo SW. Promoter hypermethylation of progesterone receptor isoform B (PR-B) in adenomyosis and its rectification by a histone deacetylase inhibitor and a demethylation agent Reprod. Sci. 17, 995-1005 (2010).

- Adenomyosis is an epigenetic disease amenable to rectification by pharmacological agents.

54. Nie J, Lu Y, Liu X, Guo SW. Immunoreactivity of progesterone receptor isoform $\mathrm{B}$, nuclear factor $\kappa \mathrm{B}$, and $\mathrm{I} \kappa \mathrm{B} \alpha$ in adenomyosis. Fertil. Steril. 92, 886-889 (2009).

55. Shen F, Liu X, Geng JG, Guo SW. Increased immunoreactivity to SLIT/ROBO1 in ovarian endometriomas: a likely constituent biomarker for recurrence. Am. J. Pathol. 175, 479-488 (2009).

56. Nie J, Liu X, Zheng Y, Geng JG, Guo SW. Increased immunoreactivity to SLIT/ROBO1 and its correlation with severity of dysmenorrhea in adenomyosis. Fertil. Steril. 95, 1164-1167 (2011).

- The SLIT/ROBO1 marker may be utilized as an index of the severity of dysmenorrhea.

57. Liu X, Nie J, Guo SW. Elevated immunoreactivity to tissue factor and its association with dysmenorrhea severity and the amount of menses in adenomyosis. Hum. Reprod. 26, 337-345 (2011).
58. Huang YQ, Zheng W, Mu L et al. Expression of tyrosine kinase receptor $\mathrm{B}$ in eutopic endometrium of women with adenomyosis. Arch. Gynecol. Obstet. 283, 775-780 (2011).

59. Campo S, Campo V, Benagiano G. Infertility and adenomyosis. Reprod. Biomed. Online 24, 35-46 (2012).

60. Hever A, Roth RB, Hevezi PA et al. Molecular characterization of human adenomyosis. Mol. Hum. Reprod. 12, 737-748 (2006).

61. Brosens I, Kung G, Benagiano G.

Is adenomyosis the neglected phenotype of an endomyometrial dysfunction syndrome? Gynecol. Surg. doi:10.1007/s10397-011-07233 (2012) (Epub ahead of print).

62. Kennedy S, Hadfield R, Westbrook C, Weeks DE, Barlow D, Golding S. Magnetic resonance imaging to assess familial risk in relatives of women with endometriosis. Lancet 352(9138), 1440-1441 (1998). 